\section{Telangiectasia macular eruptiva Perstans y síndrome de Conn}

\section{Sr. Director}

La telangiectasia macular eruptiva Perstans (TMEP) es una variedad cutánea de mastocitosis claramente diferenciada que ocasionalmente se ha asociado a neoplasias de estirpe linfoproliferativa. Referimos un caso poco frecuente y escasamente referido en la literatura de asociación con una neoplasia de estirpe sólida suprarrenal.

Presentamos el caso de una mujer de 36 años sin antecedentes personales de interés, que desde hace 6 años y coincidiendo con el último trimestre de su segundo embarazo comienza a presentar en la zona pectoral y en la raíz de los miembros inferiores, máculas eritematosas y marronáceas de aspecto telangiectásico, que se urtican tras los baños con agua caliente y muy ligeramente tras el frotamiento (signo de Darier dudoso). Mejora discretamente con la ingesta periódica de antihistamínicos de primera generación. El examen histológico reveló discreta infiltración mastocitaria en dermis superficial con presencia de capilares ectásicos en dermis papilar. Asimismo la paciente comenzó a desarrollar episodios de hipertensión de difícil control, ante lo cual se pautó tratamiento con Fosinopril $20 \mathrm{mg} /$ día acompañados de cierta debilidad y cefaleas no filiadas. La histología corroboró el diagnóstico clínico de telangiectasia macular eruptiva perstans y solicitamos estudio completo incluyendo: hemograma, bioquímica, sedimento urinario, triptasa sérica y metanefrinas urinarias y 5 hidroxi-indolacético para descartar mastocitosis sistémica y feocromocitoma o tumor carcinoide asociado. Los exámenes solicitados revelaron cifras de potasio bajas de 3,1 y 2,9 mEq/L (N: 3,5-5 mEq/L) en dos exámenes consecutivos con normalidad del resto de parámetros estudiados (la segunda cifra objetivada tras la suspensión de fosinopril).

Ante la sospecha de un hiperaldosteronismo solicitamos determinación de aldosterona (678 pg/mL; N: 10-105 pg/mL) y actividad plasmática de renina $0,3 \mathrm{ng} / \mathrm{ml}(\mathrm{N}: 0,4-2,3 \mathrm{ng} / \mathrm{ml})$ así como TAC suprarrenal bilateral que puso de manifiesto una imagen nodular hipodensa inferior a $2 \mathrm{~cm}$ de diámetro, también visualizada mediante gammagrafía (Fig. 1), con diagnóstico de adenoma suprarrenal derecho confirmado histológicamente tras la realización de una adrenalectomía unilateral por vía laparoscópica.

La telangiectasia macular eruptiva perstans (TMEP) fue descrita inicialmente por Moynahan en 1949 como una variedad cutánea de mastocitosis claramente diferenciada de las comunicadas hasta el momento. Los mastocitos suelen quedar confinados a la dermis papilar salvo cuando existe afectación sistémica y se originan a partir de las células madres pluripotenciales que expresan el antígeno CD34 y cuyo fenotipo depende de la expresión de proteínas séricas, de ahí que la determinación de triptasa sérica sea ampliamente utilizada para monitorizar clínicamente la evolución de estos enfermos.

Se ha comunicado previamente la aparición o empeoramiento de las mastocitosis cutáneas con el embarazo, lo cual sugeriría un concurso importante de los estímulos hormonales que acontecen en este estado, aunque todavía no está totalmente esclarecido (3). Por otro lado, durante estos años hemos asistido a comunicaciones punutales que relacionan esta variedad de mastocitosis con neoplasias de orden linfoproliferativo $(4,5)$. No podemos afirmar que en los casos referidos en la literatura se cumplan íntegramente los criterios expuestos por Curth (6) en 1974 y que en la actualidad siguen vigentes para definir una dermatosis como paraneoplásica, aunque dicha relación parece no ser meramente anecdótica. Recientemente se han descrito

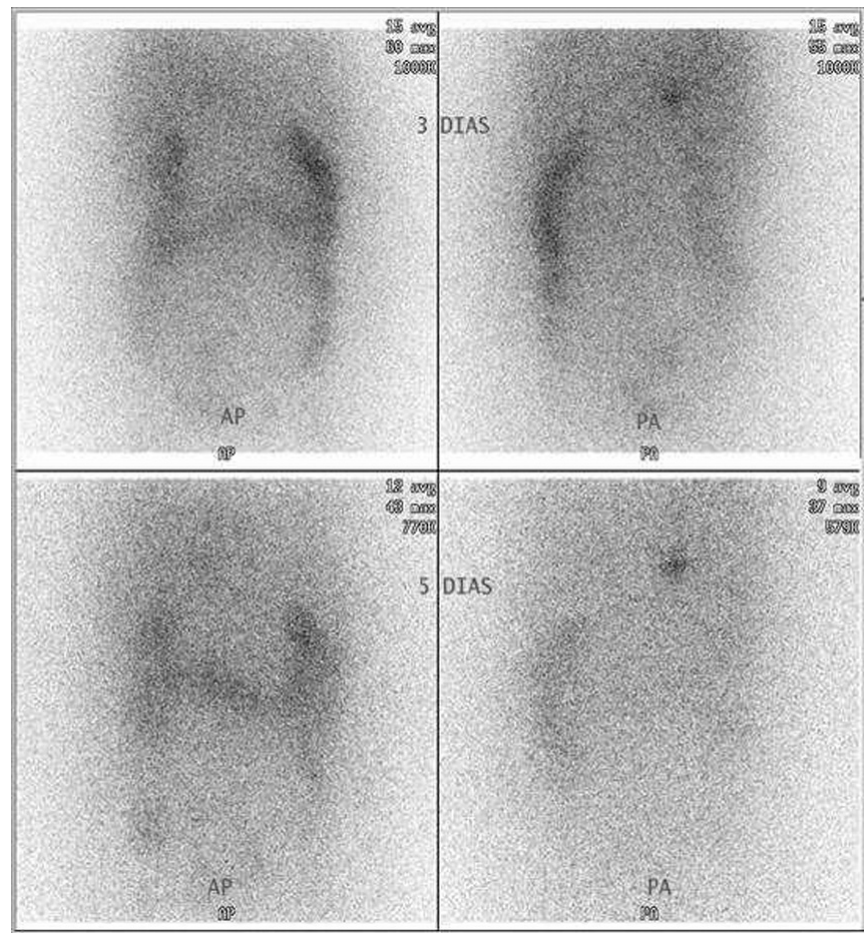

Fig. 1. Adenoma suprarrenal derecho (gammagrafía suprarrenal).

neoplasias de estirpe sólida relacionadas con la TMEP (7), por lo que, si bien, el significado de la asociación en nuestro caso es incierto, sí requiere tener una actitud más cuidadosa ante este tipo de pacientes.

\section{R. Ruiz Villaverde, I. Hernández Jurado, D. Sánchez Cano ${ }^{1}$}

Unidad de Dermatología. Hospital de Poniente. El Ejido. Almería. ${ }^{I}$ Servicio de Medicina Interna. Hospital Clínico Universitario. Granada

1. Moynahan EJ. Urticaria pigmentosa (telangiectasia macularis eruptiva perstans). Proc R Soc Med 1949; 42: 346-347.

2. Tebbe B, Stavropoulos PG, Krasagakis K, et al. Cutaneous mastocytosis in adults. Evaluation of 14 patients with respect to systemic disease manifestations. Dermatology 1998; 197: 101-108.

3. Donahue JG, Lupton JB, Golichowski AM. Cutaneous mastocytosis complicating pregnancy. Obstet Gynecol 1995; 85: 813-5.

4. Martin LK, Romanelli P, Ahn YS, Kirsner RS.Telangiectasia macularis eruptiva perstans with an associated myeloproliferative disorder. Int $\mathbf{J}$ Dermatol 2004; 43: 922-4.

5. Bachmeyer C, Guillemette J, Blum L, Turc Y, Dhote R, Fermand JP, Aractingi S. Telangiectasia macularis eruptiva perstans and multiple myeloma. J Am Acad Dermatol 2000; 43: 972-4.

6. Curth HO. A spectrum of organ systems that respond to the presence of cancer. How and why the skin reacts. Ann N Y Acad Sci 1974; 230: 43542.

7. Pascual JC, Banuls J, Albares MP, Vergara G, Belinchon I, Silvestre JF, et al. Presentation of telangiectasia macularis eruptiva perstans as a longstanding solitary plaque associated with renal carcinoma. J Cutan Med Surg 2003; 7: 399-402. 